Laser Chem., 1999, Vol. 19, pp. 271-274

Reprints available directly from the publisher Photocopying permitted by license only
(C) 1999 OPA (Overseas Publishers Association) N.V. Published by license under the Harwood Academic Publishers imprint, part of The Gordon and Breach Publishing Group.

\title{
TIME-RESOLVED RESONANCE RAMAN SPECTROSCOPY OF EXCITED-STATE PORPHYRINS
}

\author{
S. E. J. BELL ${ }^{a}$, , J. H. RICE ${ }^{\text {a }}$, J. J. MCGARVEY ${ }^{\text {a }}$, \\ R. E. HESTER ${ }^{b}$, J. N. MOORE ${ }^{\text {b }}$, R. N. PERUTZ ${ }^{\text {b }}$, T.-Q. YE ${ }^{\text {, }}$, \\ Y. MIZUTANI ${ }^{\mathrm{c}}$ and T. KITAGAWA ${ }^{\mathrm{c}}$ \\ ${ }^{a}$ School of Chemistry, The Queen's University of Belfast, \\ Belfast BT9 5AG, UK, \\ ${ }^{\mathrm{b}}$ Dept. of Chemistry, University of York, Heslington, York Y01 5DD, UK; \\ ${ }^{\mathrm{c}}$ Institute for Molecular Science, Okazaki National Research Institutes, \\ Myodaiji, Okazaki 444, Japan
}

\section{(Received 11 April 1997)}

Time-resolved resonance Raman $\left(\mathrm{TR}^{3}\right)$ and absorbance difference studies of the excited states of $\mathrm{Cu}$ (TPP) (TPP $=5,10,15,20$-tetraphenylporphyrin) have been carried out with $<10$ ps times resolution in THF and pyridine solvents. In THF the distinctive transient Raman bands in the $\nu_{2}$ and $\nu_{4}$ regions, previously observed with ns laser pulses, grow in the first $55 \mathrm{ps}$ before decaying in 100's of ps. The $\Delta \mathrm{A}$ spectra also show biphasic decay. This behaviour is associated with attack by solvent on the 4-coordinate excited state to form the longer lived species observed in $\mathrm{TR}^{3}$ experiments.

In pyridine two component decay is also observed but it is the shorter-lived species which gives the transient Raman bands seen previously with ns laser excitation. This state is different from that seen in THF. At 5 ps delay $\nu_{4}$ is broader than in the ground state and, more importantly, there is a significant shift in the two pyridine bands at $c a$. $1000 \mathrm{~cm}^{-1}$. This implies a significant involvement of the pyridine-based orbitals in the excited state.

Keywords: Porphyrins; metalloporphyrins; excited; Raman; time-resolved; photochemistry

Time-resolved resonance Raman studies of the excited states of porphyrins have now become established as an excellent means of characterising the electronic structure of such species [1]. Metallopor-

\footnotetext{
* Corresponding author.
} 
phyrins with closed-shell metals have a relatively simple photochemistry with well-defined singlet and triplet excited states but in $\mathrm{Cu}$ (II) porphyrins additional $d-d$ and charge-transfer (LMCT and MLCT) excited states [3-5] now lie close in energy to the porphyrin $\pi-\pi^{*}$ states [2]. The relative energies of all these states depend strongly on the solvent, which can coordinate the $\mathrm{Cu}$ centre in either the ground or excited states. $\mathrm{Cu}$ (TPP) in THF gives distinctive transient Raman spectra, when excited with ns laser pulses at wavelengths $415-450 \mathrm{~nm}$. These spectra typically show double peaks in the $\nu_{2}$ and $\nu_{4}$ regions, one set of which are in positions close to those of the ground state species and a second major pair which are shifted $c a .20 \mathrm{~cm}^{-1}$ lower and are due to an excited 5-coordinate $\mathrm{Cu}$ (TPP)-THF complex [3-5].

The $\Delta A$ spectra of $\mathrm{Cu}(\mathrm{TPP})$ in THF recorded at 5 and $100 \mathrm{ps}$ (Fig. 1 , insert) show two components, one of which maximises near the Soret band and one which is farther to the red. The latter band (which decays by $100 \mathrm{ps}$ ) is very similar to that of simple triplet $\mathrm{Cu}$ (TPP). The former absorption band is associated with the species giving rise to the

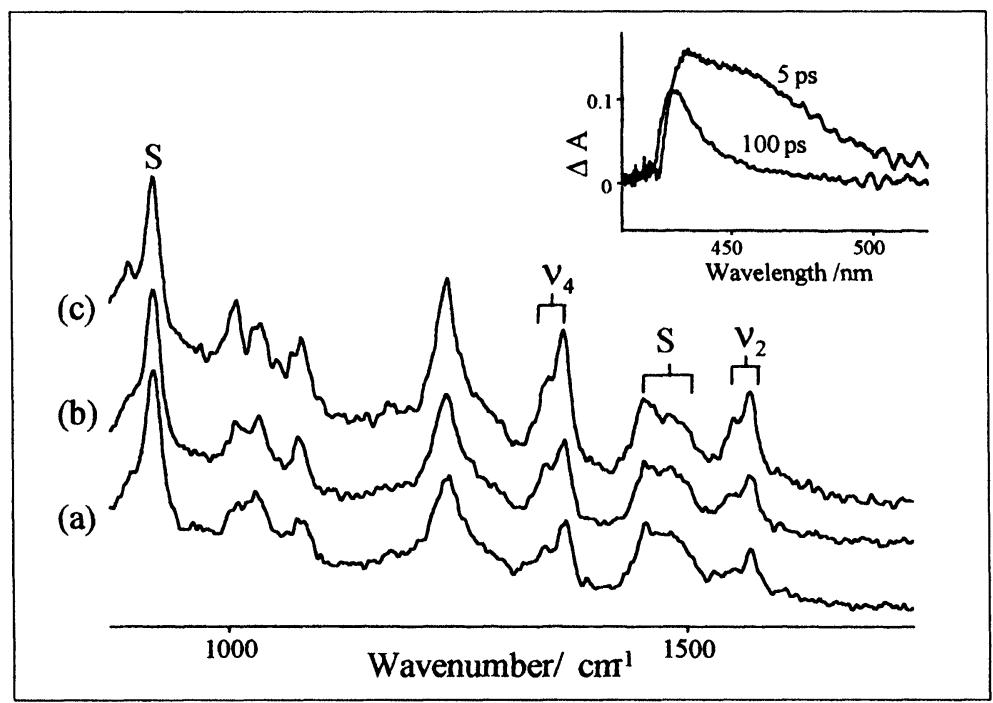

FIGURE 1 Time-resolved resonance Raman spectra of $\mathrm{Cu}$ (TPP) in THF. $\lambda_{\text {pump }}=540 \mathrm{~nm}, \lambda_{\text {probe }}=410 \mathrm{~nm}$. Pump-probe time delay: (a) $10 \mathrm{ps}$; (b) $25 \mathrm{ps;} \mathrm{(c)}$ $55 \mathrm{ps}$. The larger solvent bands are labelled $\mathrm{S}$. Insert- Transient absorbance difference spectra of $\mathrm{Cu}(\mathrm{TPP})$ in THF at $5 \mathrm{ps}$ and $100 \mathrm{ps}$. 
distinctive transient Raman bands. $\mathrm{TR}^{3}$ measurements also show evidence for biphasic decay. Loss of the transient bands occurs on the timescale of hundreds of ps but spectra taken at short (10-55 ps) delays (Fig. 1), show that, although both the transient and residual ground state bands are present at $10 \mathrm{ps,} \mathrm{both} \mathrm{these} \mathrm{features} \mathrm{grow} \mathrm{in}$ intensity up to $55 \mathrm{ps}$, after which the transient bands decay. At $10 \mathrm{ps}$ some of the excited $\mathrm{Cu}$ (TPP) has not yet undergone solvent attack and so is still in the excited triplet state which can be observed in the $\Delta A$ spectra but is Raman silent at this probe wavelength. In the next 45 ps solvent attack proceeds faster than decay of either the triplet state or the 5-coordinate excited exciplex so that the population of the 5coordinate exciplex rises before ultimately decaying in 100's of ps.

With pridine solvent the excited state lifetime is significantly shorter than in THF but again the $\Delta A$ spectra at 5 ps show a double feature. However, in this case the rate of decay of the 5-coordinate species is faster than that of solvent attack on the 4-coordinate porphyrin triplet present, so that it is the shorter wavelength feature which decays first in the $\Delta A$ spectra. The nature of this short lived 5-coordinate state is clearly different from that observed in THF. The data in Figure 2 show

(d)
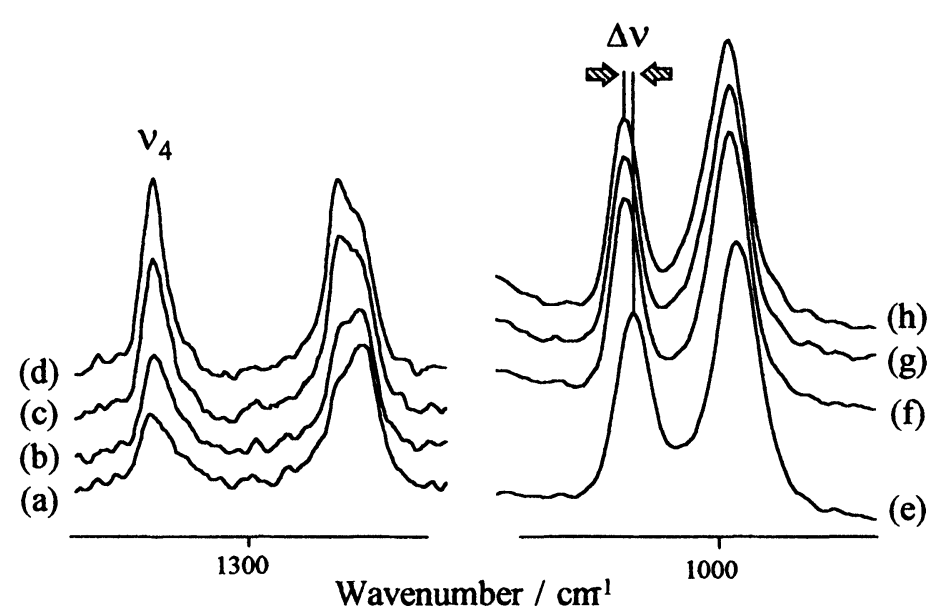

FIGURE 2 Time-resolved resonance Raman spectra of $\mathrm{Cu}$ (TPP) in pyridine. Two spectral regions are shown. $\lambda_{\text {pump }}=540 \mathrm{~nm}, \lambda_{\text {probe }}=410 \mathrm{~nm}$. Pump-probe time delay: (a) and (e) $5 \mathrm{ps}$; (b) and (f) $15 \mathrm{ps;} \mathrm{(c)} \mathrm{and} \mathrm{(g)} 25 \mathrm{ps}$; (d) and (h) $35 \mathrm{ps}$. 
that at the shortest time delay $\nu_{4}$ is broader than in the ground state (which is consistent with literature data $[4,5]$ ) but the most important observation for structural assignment of the state is that in the spectrum recorded at $5 \mathrm{ps}$ there is a significant shift in the two pyridine bands at $c a .1000 \mathrm{~cm}^{-1}$ compared to their positions in the spectra recorded at longer time delays and those of the ground state. This implies a significant involvement of the pyridine-based orbitals in the excited state.

\section{References}

[1] Bell, S. E. J. (1996). Analyst, 121, 107R.

[2] Asano-Someda, M., Sato, S., Aoyagi, K. and Kitagawa, T. (1995). J. Phys. Chem., 99, 13800.

[3] Kruglik, S. G., Apanasevich, P. A., Chirvony, V. S., Kvach, V. V. and Orlovich, V. A. (1995). J. Phys. Chem., 99, 2978.

[4] de Paula, J. C., Walters, V. A., Jackson, B. A. and Cardozo, K. (1995). J. Phys. Chem., 99, 4373.

[5] Jeoung, S. C., Kim, D., Cho, D. W. and Yoon, M. (1995). J. Phys. Chem., 99, 5826. 